\title{
The combined impact of persistent infections and human genetic variation on C-reactive protein levels
}

\author{
F. Hodel ${ }^{1,2}$, O. Naret ${ }^{1,2}$, C. Bonnet ${ }^{1,2}$, N. Brenner ${ }^{3}$, N. Bender ${ }^{3}$, T. Waterboer ${ }^{3}$, P. Marques-Vidal ${ }^{4}$, P. Vollenweider ${ }^{4}$, and J. \\ Fellay ${ }^{1,2,5, \square}$ \\ ${ }^{1}$ Global Health Institute, School of Life Sciences, EPFL, Lausanne, Switzerland \\ ${ }^{2}$ Swiss Institute of Bioinformatics, Lausanne, Switzerland \\ ${ }^{3}$ Division of Infections and Cancer Epidemiology, German Cancer Research Center, Heidelberg, Germany \\ ${ }^{4}$ Department of Medicine, Internal medicine, Lausanne University Hospital and University of Lausanne, Lausanne, Switzerland \\ ${ }^{5}$ Precision Medicine Unit, Lausanne University Hospital and University of Lausanne, Lausanne, Switzerland
}

\begin{abstract}
Multiple human pathogens establish chronic, sometimes lifelong infections. Even if they are often latent, these infections can trigger some degree of local or systemic immune response, resulting in chronic low-grade inflammation. There remains an incomplete understanding of the potential contribution of both persistent infections and human genetic variation on chronic low-grade inflammation. We searched for potential associations between seropositivity for 13 persistent pathogens and the plasma levels of the inflammatory biomarker C-reactive protein (CRP), using data collected in the context of the UK Biobank and the CoLaus|PsyCoLaus Study, two large population-based cohorts. We performed backward stepwise regression starting with the following potential predictors: serostatus for each pathogen, polygenic risk score for CRP, as well as demographic and clinical factors known to be associated with CRP. We found evidence for an association between Chlamydia trachomatis (Pvalue $=5.04 \mathrm{e}-3)$ and Helicobacter pylori $(\mathrm{P}$-value $=8.63 \mathrm{e}-4)$ seropositivity and higher plasma levels of CRP. We also found an association between pathogen burden and CRP levels (P-value $=4.12 \mathrm{e}-4)$. These results improve our understanding of the relationship between persistent infections and chronic inflammation, an important determinant of long-term morbidity in humans.
\end{abstract}

human genomics | persistent infections | inflammation | c-reactive protein Correspondence: jacques.fellay@epfl.ch

\section{Introduction}

Inflammation is a complex and necessary response of the immune system to harmful stimuli such as tissue injury, infection, or exposure to toxins (1). During the acute phase that is characterized by blood flow changes and increased blood vessel permeability, plasma proteins and leukocytes migrate from the circulation to the site of inflammation (2). This immediate protective response usually enables the elimination of the initial cause of the cell injury and the restoration of homeostasis. However, when the acute response fails to clear tissue damage, for example because of prolonged exposure to stimuli, inflammation can become a chronic process (3). A number of common diseases are at least partly caused by chronic inflammation, including coronary artery disease, type 2 diabetes, and some cancers (4). Thus, although inflammation plays an important role in human defense against aggres- sion, it also contributes to the pathophysiology of multiple diseases of major public health importance.

Diagnostic tests are capable of detecting the presence and intensity of systemic inflammation (5). The most commonly used inflammatory biomarker is the acute phase reactant $\mathrm{C}$ reactive protein (CRP). This ring-shaped protein is produced by hepatocytes upon stimulation by pro-inflammatory cytokines such as interleukin (IL)-1 $1 \beta$, IL-6, and TNF- $\alpha$.

Although CRP is commonly used as a sensitive indicator of inflammation, the factors influencing its baseline plasma levels are only partially understood. Circulating amounts of CRP are positively associated with age, body mass index (BMI) and smoking, and inversely with male sex and physical activity (6-8). In addition, large-scale genomic analyses have shown that common genetic variation explains up to $7.0 \%$ of the variance in plasma CRP levels (9).

To get a more comprehensive view of the factors influencing chronic inflammation in the general population, we used samples and data from the UK Biobank and the CoLaus|PsyCoLaus study to search for associations between baseline CRP levels and chronic infection by persistent/latent pathogens, after careful adjustment for all known demographic, clinical and genomic influences. Indeed, some infectious agents causing long-term infections in humans have been shown to trigger some degree of local or systemic immune response, resulting in a chronic state of low-grade inflammation that may lead to deleterious health outcomes $(10,11)$.

\section{Methods}

Study cohorts. The UK Biobank is a population-based exploratory study of which the enrollment procedure has been outlined previously (12). In brief, half a million men and women between the ages of 40 and 69 (45.6\% male, mean age \pm SD: $56.5 \pm 8.1$ ) visited one of 22 UK Biobank screening centers in England, Scotland and Wales between 2006 and 2010. The evaluation included a survey, a personal interview, and a number of physical measurements and blood. Urine and saliva samples were also collected for long-term storage. This research was undertaken with approved access NOTE: This preprint reports new research that has not been certified by peer review and should not be used to guide clinical practice. 
to UK Biobank data under application number 50085 (PI: Fellay). All UK Biobank study participants gave informed consent at the time of recruitment. Ethical approval for the UK Biobank study was obtained from the North West Centre for Research Ethics Committee (11/NW/0382).

The CoLaus|PsyCoLaus study is a prospective populationbased study initiated in 2003 in Lausanne, Switzerland (www.colaus-psycolaus.ch) (13). It involves more than 6'000 participants of European ancestry ( $47.5 \%$ male) initially aged 35 to 75 years (mean \pm SD: $51.1 \pm 10.9$ ), thus representing a sample of approximately $10 \%$ of the inhabitants of Lausanne. Individuals were randomly recruited from the general population and are monitored every 5 years regarding their lifestyle and health status. Detailed phenotypic information was obtained from each study participant through questionnaires, physical assessment, and biological measurements of blood and urine markers. The institutional Ethics Committee of the University of Lausanne, which afterward became the Ethics Commission of Canton Vaud (www.cervd.ch) approved the baseline CoLaus|PsyCoLaus study (reference 16/03, decisions of 13th January and 10th February 2003), and written consent was obtained from all participants.

DNA genotyping and quality checks. Genotyping and imputation of UK Biobank individuals have been fully described by Bycroft et al. (14). Briefly, samples were genotyped on either the UK BiLEVE Axiom array (Affymetrix) or UK Biobank Axiom array (Applied Biosystems). Genotypes were phased using SHAPEIT3 and the 1000 Genome phase 3 dataset as a reference, then imputed using IMPUTE4 using the Haplotype Reference Consortium data, 1000 Genomes phase 3, and UK10K data as references (15-17). Post-imputation quality checks resulted in a total number of 9'349'624 single nucleotide polymorphisms (SNPs) available for analyses. DNA samples from 5'399 CoLauslPsyCoLaus participants were genotyped for 799'653 SNPs using the BB2 GSK-customized Affymetrix Axiom Biobank array. Quality control procedures and imputation of genotypes have been previously described in Hodel et al. (18). A total of 9'031'263 SNPs from the CoLaus|PsyCoLaus dataset were included for further analyses (flowchart of inclusion/exclusion criteria in Supplementary Fig. 1).

Measurement of inflammatory biomarkers. For the UK Biobank, non-fasting venous blood samples $(\sim 50 \mathrm{~mL})$ were collected at recruitment. Blood samples were shipped at $4{ }^{\circ} \mathrm{C}$ to the central processing and archiving facility in Stockport. Serum high-sensitivity CRP (hs-CRP) concentrations were measured in participants by immunoturbidimetric assay on a Beckman Coulter AU5800. The manufacturer's analytical range was 0.08 to $80 \mathrm{mg} / \mathrm{L}$. Ninety-five individuals with a hsCRP level $20 \mathrm{mg} / \mathrm{L}$ were removed from the analysis. For CoLauslPsyCoLaus, venous blood samples $(\sim 50 \mathrm{~mL})$ were drawn in the fasting state and allowed to clot. Serum blood samples were kept at $80^{\circ} \mathrm{C}$ before assessment of cytokines and sent in dry ice to the laboratory. Hs-CRP was assessed by immunoassay and latex HS (IMMULITE 1000-High, Diagnostic Products Corporation, LA, CA, USA). For quality control, repeated measurements were conducted in 80 subjects randomly drawn from the initial sample. Forty-seven individuals with hs-CRP levels above $20 \mathrm{mg} / \mathrm{L}$ were assigned a value of 20 by the manufacturer and were therefore removed from the hs-CRP analyses as they are indicative of acute inflammation.

Serological analyses. To assess humoral responses to a total of 56 antigens derived from 24 persistent infectious agents (45 antigens from 20 pathogens in UK Biobank, and 38 antigens from 18 pathogens in CoLaus(PsyCoLaus), serum samples were independently analyzed by the Infections and Cancer Epidemiology Division at the German Cancer Research Center (Deutsches Krebsforschungszentrum, DKFZ) in Heidelberg $(19,20)$. Seroreactivity was measured at serum dilution 1:1000 using multiplex serology based on glutathioneS-transferase (GST) fusion capture immunosorbent assays combined with fluorescent bead technology. For each infectious agent tested, antibody responses were measured for one to six antigens and then expressed as a binary result (IgG positive or negative) based on predefined median fluorescence intensity (MFI) thresholds (21). For our analysis, only antigens shared between the two cohorts were retained, resulting in a final combination of 27 antigens from 13 pathogens. To define overall seropositivity against infectious agents when more than one antigen was used, we applied the pathogenspecific algorithms suggested by the manufacturer. Details of the methods on how the antigens were combined have been described previously (21).

Combining study cohorts. Upon completion of the genotyping and quality control (QC) analyses for each cohort, imputed datasets were matched on strand, SNP ID and genomic coordinates. Additional analyses and QC checkpoints were performed to ensure proper merging. This resulted in a dataset of 12'055 unique individuals of European ancestry and a total of 6'899'629 markers.

Genome-wide association study of hs-CRP and tissue prioritization. Single-marker genome-wide association study (GWAS) of log-10 transformed hs-CRP was conducted using a general linear model association analysis in PLINK 2.0 , adjusting for sex, age, BMI, and the top 10 principal components (PCs) of the genotyping data (22). Some individuals were excluded at this stage due to missing covariate data and GWAS was thus performed on a final set of 12'031 individuals. We performed differentially expressed gene (DEG) analysis using the GENE2FUNC procedure in FUMA (23). FUMA defined the DEGs in each tissue by performing a twosided Student's t-test for each tissue against the remaining tissues. Each of the 30 general tissue types available from GTEx v8 was tested for up-regulation, down-regulation, and both-sided DEG sets (24). Genes with a P-value $<0.05$ and an absolute fold change $\geq 1.5$ (0.58 in $\log 2$ scale) were defined as DEG. 
Polygenic risk score calculation for hs-CRP level. We carried out a polygenic risk score (PRS) analysis to investigate the relationship between human genetic variation and hs-CRP levels. A CRP-PRS was calculated for each study participant based on the risk effects of common SNPs derived from GWAS summary statistics of hs-CRP. As a baseline cohort, we referred to the GWAS summary statistics of the CHARGE cohort $\left(\mathrm{N}=204^{\prime} 402\right.$, heritability $\left.\mathrm{h} 2=6.5 \%\right)$ $(9,25)$. These summary statistics were used to construct the CRP-PRS in our target cohort consisting of the merged UK Biobank and CoLausIPsyCoLaus data using the clumping and thresholding method of the PRSice-2 v2.2.7 software (26). We used a standardized method to obtain PRS, by multiplying the dosage of risk alleles for each variant by the effect size in the GWAS and summing the scores across all of the selected variants. SNPs were clumped based on linkage disequilibrium (LD) $(\mathrm{r} 2 \geq 0.1)$ within a $250 \mathrm{~kb}$ window. Model estimates of the PRS effect were adjusted for sex, age, BMI, and the top 10 PCs. As an additional quality control, the distribution of PRS was checked in each cohort separately, to ensure that they followed a normal distribution.

Analyses of the determinants of hs-CRP levels. We used linear regression with backward selection to identify the factors significantly associated with hs-CRP plasma levels. Tested covariates included serostatus for each pathogen, polygenic risk score for CRP, age, sex, BMI, and the first 10 PCs of the genotyping data. P-value $<0.05$ was considered statistically significant. The analysis was performed using the stepAIC function in R version 4.0.5 (27).

\section{Results}

Baseline characteristics of study participants. We studied a total of 12'055 individuals with available hs-CRP level, serological results, and genome-wide genotyping data from two independent population-based studies: the UK Biobank $\left(\mathrm{N}=8^{\prime} 371\right)$ and the CoLaus/PsyCoLaus study $\left(\mathrm{N}=3^{\prime} 684\right)$ (Supplementary Fig. 1). Participants ranged in age from 35 to 75 years (mean age \pm SD: $55.68 \pm 9.07$ ), with a majority of women $(55.4 \%)$ and a mean BMI of 26.80 ( \pm SD: 4.73). HsCRP level was measured in all participants. The median hsCRP level was $1.30 \mathrm{mg} / \mathrm{L}$ (10th, 90th percentiles: $0.35 \mathrm{mg} / \mathrm{L}$, $5.10 \mathrm{mg} / \mathrm{L}$ ). Fig. 1 shows the distributions of age, sex, BMI and $\log 10$-transformed hs-CRP in both cohorts. We observed a very comparable distribution of all relevant variables in the two cohorts, which were merged for downstream analyses. Supplementary Fig. 2 shows associations of hs-CRP with demographic and clinical factors. Higher levels of hs-CRP associated with female sex, age and increased BMI (P-values $=1.5 \mathrm{e}-3,3.4 \mathrm{e}-69$, and $\approx 0$, respectively).

The impact of genetic variation on hs-CRP levels. The filtered genetic variants from the two cohorts were combined (see Methods) to increase sample size. To estimate sample variation, and to control for potential population structure and genotyping bias, principal component analysis (PCA) was performed using the correlation matrix of the genotyp-
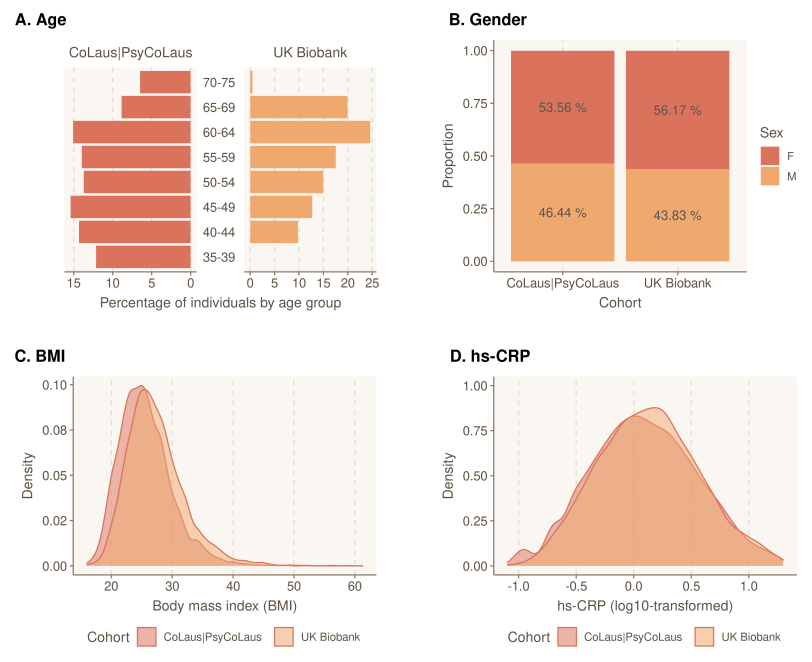

Fig. 1. Baseline characteristics of the study cohort. Distribution of $A$ ) age, $B$ ) gender, $C$ ) body mass index (BMI) and $D$ ) hs-CRP for participants by subcohort.

ing data. PCA plots for the first ten principal components (PC1-PC10) are shown in Supplementary Fig. 3A, annotated by the original cohort from which the sample was drawn. We observed that samples from both subgroups (UK Biobank and CoLauslPsyCoLaus) were segregated on the first PC (PC1) and eighth PC (PC8), but not on the other PCs. The top 10 PCs explained $61 \%$ of the total variance and were used throughout the study to correct for stratification (Supplementary Fig. 3B).

We performed GWAS in our merged data using a continuous, quantitative approach to search for human genetic determinants of hs-CRP levels. Factors associated with hs-CRP levels (age, sex, and BMI) were included as covariates in the linear regression models, in addition to the coordinates of the top 10 PCs (Supplementary Fig. 2). The Manhattan plot for the GWAS results is presented in Fig. 2. Our study replicates six previously reported associated loci involved in immune and hepatic metabolic pathways, including rs 4655584 in $L E P R(\mathrm{P}$-value $=5.57 \mathrm{e}-27)$, rs4129267 in IL6R $(\mathrm{P}$-value $=$ 4.23e-19), rs 2794520 in $C R P(\mathrm{P}-$ value $=2.51 \mathrm{e}-32)$, rs 780093 in $G C K R$ (P-value $=1.85 \mathrm{e}-13)$, rs7970695 in HNF1A (Pvalue $=2.32 \mathrm{e}-29)$ and $\mathrm{rs} 429358$ in the $A P O E$ locus $(\mathrm{P}$-value $=3.30 \mathrm{e}-51)(9,28-31)$. Quantile-quantile $(\mathrm{Q}-\mathrm{Q})$ plot and genomic control lambda $(\lambda=1.03)$ revealed no deviation in the test statistics compared with the expectation (Supplementary Fig. 4). We then performed differential expression analysis (DEA) across GTEx v8 RNA-seq data for 30 general tissue types on the mapped GWAS-associated genes and identified a significant enrichment of upregulated genes in human liver (Supplementary Fig. 5).

Next, we computed a CRP-PRS to investigate the effect of multiple gene variants on hs-CRP levels. A total of 1'809 SNPs were included at the best $\mathrm{P}$-value threshold (P-value $=3.65 \mathrm{e}-3$ ). The PRS followed a normal distribution in the merged cohort, as well as in each subcohort separately (Supplementary Fig. 6). To describe the influence of common 


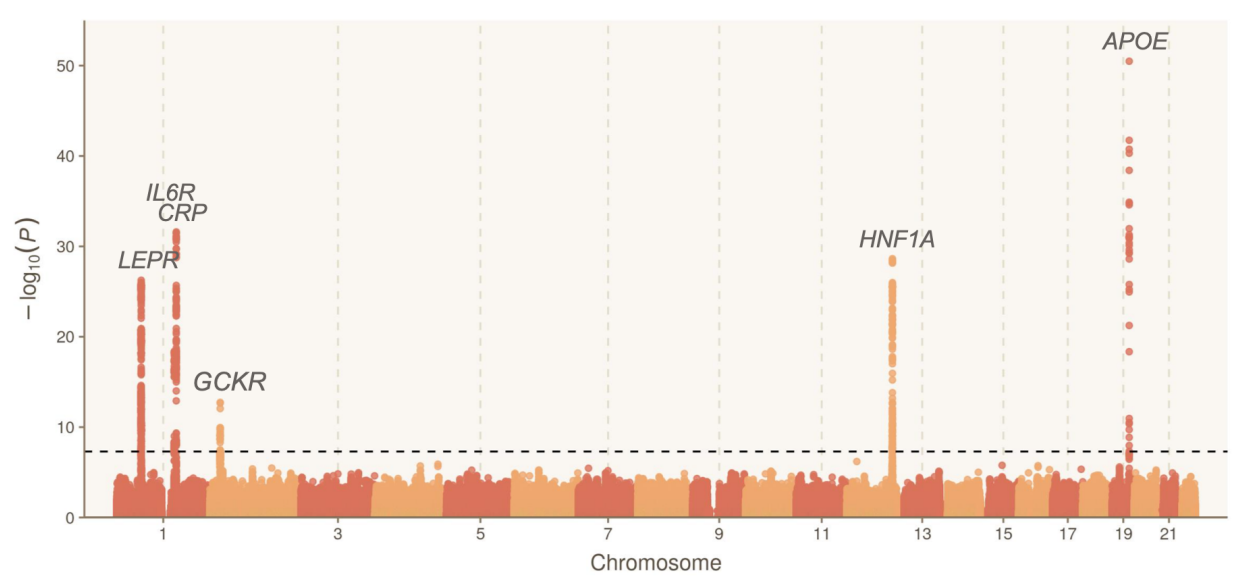

Fig. 2. Manhattan plot of genome-wide association results of hs-CRP levels. Manhattan plot showing the significance of association of all SNPs across chromosomes 1 to 22. SNPs are plotted on the x-axis according to their physical position on each chromosome and the strength of the association with serostatus is indicated on the $y$-axis as -log10 P-value. The dashed line marks the Bonferronicorrected genome-wide significance threshold of $5 \mathrm{e}-08$. Gene labels are annotated as the nearby genes to the significant SNPs.

human genetic variation on plasma hs-CRP levels, we quantified the trait variance (R2) explained by the derived PRS and covariates across individuals. We observed that the variance explained by the full model was $25.8 \%$, with $21.5 \%$ attributed to the demographic and clinical covariates and $4.3 \%$ to the CRP-PRS. The association between the CRP-PRS and hs-CRP levels was very strong $(\mathrm{P}$-value $=6.58 \mathrm{e}-123$; Supplementary Fig. 7), with hs-CRP levels increasing by 0.48 [Standard error (SE) 0.02] for each standard deviation increment in CRP-PRS.

Associations between persistent/latent infections and hs-CRP levels. We searched for associations between hsCRP levels and serostatus for the following persistent or frequently recurring human pathogens: 10 viruses (BK virus (BKV), Cytomegalovirus (CMV), Epstein-Barr virus (EBV), Human Herpes Virus (HHV)-6, HHV-7, Herpes Simplex Virus (HSV)-1, HSV-2, JC virus (JCV), Kaposi's sarcomaassociated herpesvirus (KSHV) and Varicella zoster virus (VZV)); two bacteria (Chlamydia trachomatis (C. trachomatis) and Helicobacter pylori (H. pylori)); and one parasite (Toxoplasma gondii (T. gondii)) (Fig. 3). The overall seropositivity ranged from $6.57 \%$ (KSHV) to $95.25 \%$ (EBV). Cohort-separated seroprevalences are shown in Supplementary Fig. 8 and 9.

Using backward stepwise regression, we observed associations of hs-CRP levels with seropositivity for $H$. pylori ( $\mathrm{P}$ value $=8.63 \mathrm{e}-4)$ and $C$. trachomatis $(\mathrm{P}$-value $=5.04 \mathrm{e}-3)$ (Table 1). The final regression model including these two pathogens, sex, age, BMI, and the top $10 \mathrm{PCs}$, explained $25.9 \%$ of the variance of hs-CRP levels. This explained fraction of the variance is similar to the value obtained without including the serological results (above), indicating that the impact of $H$. pylori and $C$. trachomatis seropositivity on chronic inflammation, even if statistically significant, is likely to be minimal at the population level.

Pathogen burden associates with higher hs-CRP levels. We then checked if the overall burden of chronic infec-

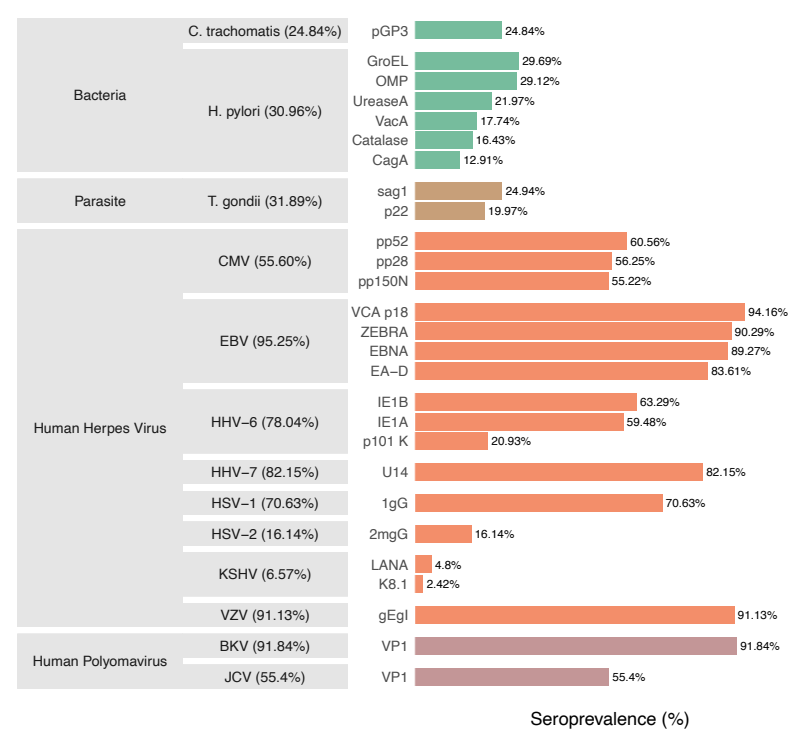

Fig. 3. Overall pathogen seropositivity and seroprevalence of tested antigens. List of the 13 pathogens and 27 antigens available from the combined study. The grey boxes indicate the pathogen on which the antigen protein is found, and the family to which the pathogen belongs. Percentages in parentheses after pathogen names indicate overall seropositivity for the specified pathogen. The percentages on the right indicate the seroprevalence of antibodies against infectious disease antigens tested using Multiplex Serology platform. For study-based figure see Supplementary Fig. 8 and 9. 
Table 1. Linear regression analysis results for hs-CRP.

\begin{tabular}{llll}
\hline Characteristic & Beta & $\mathbf{9 5 \%} \mathbf{C I}^{\mathbf{1}}$ & P-value \\
\hline C. trachomatis & & & 0.005 \\
Seronegative & - & - & \\
Seropositive & 0.02 & $0.01,0.04$ & \\
H. pylori & & & $<0.001$ \\
$\quad$ Seronegative & - & - & \\
Seropositive & 0.03 & $0.01,0.04$ & \\
Age & 0.01 & $0.01,0.04$ & $<0.001$ \\
BMI & 0.04 & $0.04,0.04$ & $<0.001$ \\
Sex & & & $<0.001$ \\
M & - & - & \\
F & 0.07 & $0.06,0.09$ & \\
CRP-PRS & 0.10 & $0.09,0.10$ & $<0.001$ \\
PC1 & 2.0 & $1.1,3.0$ & $<0.001$ \\
PC2 & -1.1 & $-1.9,-0.27$ & 0.008 \\
PC3 & 2.3 & $1.5,3.1$ & $<0.001$ \\
PC4 & -1.5 & $-2.3,-0.72$ & $<0.001$ \\
PC7 & -1.4 & $-2.2,-0.60$ & $<0.001$ \\
PC8 & 1.0 & $-0.02,2.0$ & 0.054 \\
\hline CI $=$ Confidence Interval &
\end{tabular}

tions contributes to increased hs-CRP levels. Study participants were stratified according to their overall seropositivity index, calculated by summing the number of pathogens for which they were seropositive (range: 0-13). The number of individuals in each serological stratum ranged from 5 (index $=0$ ) to 2'717 (index =7) and is presented in Fig. 4. We used a linear model to search for an association between pathogen burden and hs-CRP levels. Hs-CRP levels were found to be significantly and positively associated with increasing pathogen burden $(\mathrm{P}$-value $=4.12 \mathrm{e}-4)(\mathrm{Fig} .4)$.

\section{Discussion}

Mounting evidence suggests that exposure to multiple pathogens, even when they do not cause obvious disease, can affect the immune system and human health $(18,32,33)$. In an effort to better understand the variability of humoral immune response and inflammation patterns in response to pathogen exposure, we selected 27 antigens from 13 persistent infectious agents, which we evaluated using multiplex serology to detect specific immunoglobulin $\mathrm{G}$ levels in two well-characterized population-based cohorts.

We first studied the human genetic determinants of hs-CRP levels in our study population. Using a GWAS approach, we confirmed previously reported associations with hs-CRP levels at the CRP, LEPR, IL6R, GCKR, APOE and HNFIA-ASI loci $(9,28-31)$. These genes and genomic loci are enriched in hepatic, immune and metabolic pathways. We then investigated the relationship between common genetic variation and hs-CRP levels by calculating a PRS for all study participants. The PRS explained about $4 \%$ of the variation in hs-CRP levels, in agreement with previously published results (28). We also found that BMI was the major non-genetic predictor of hs-CRP, with approximately $19 \%$ of the variance explained.
Next, we studied the impact of persistent infections on chronic inflammation after adjustment for known influencing factors, including age, sex, BMI, and human genetic variability, as explored above. We observed an association between increased levels of hs-CRP and seropositivity for $C$. trachomatis and $H$. pylori. The two gram-negative bacteria C. trachomatis and H. pylori do not cause life-long, latent infections. Nevertheless, they are responsible for some of the most frequent chronic infections in humans.

H. pylori can colonize the gastric epithelium for long periods of time, leading to chronic inflammation of the gastric mucosa. Even if the majority of individuals infected with $H$. pylori have no symptoms, the bacterium has been causally linked with gastritis, gastric ulcer and an increased risk of gastric cancer $(34,35)$. Our results suggest a systemic impact of chronic $H$. pylori infection beyond the known local inflammatory effect on the gastric mucosa, confirming an observation made previously in a cross-sectional population-based study (36).

C. trachomatis causes genital and ocular infection. The ocular manifestation of the infection, trachoma, is the world's leading cause of preventable blindness and is endemic in many developing countries. This clinical presentation is however highly unlikely to contribute to the $25 \%$ seroprevalence of anti-Chlamydia antibodies detected in the Swiss and UK cohorts included in our study. More relevant here, C. trachomatis is the etiological agent of human chlamydia urogenital tract infection, which is the most common bacterial sexually transmitted disease. Chronic or recurrent forms of the disease are frequently observed. To our knowledge, no study has examined the direct association between $C$. trachomatis infection and hs-CRP levels at the population level. However, studies conducted in the context of associations between $C$. trachomatis and tubal factor-related subfertility and preterm delivery have also shown elevated hs-CRP levels (37-39).

Altogether these results confirm the role of chronic or recurrent bacterial infections in low-grade inflammation, reflected by a small but consistent increase in hs-CRP levels in seropositive individuals. In addition, we found an association between increased pathogen burden and hs-CRP levels by stratifying individuals according to their cumulative number of positive serological results. This indicates that latent infections might play an enhancing role on chronic low-grade inflammation, even if that effect is too small to be detected at the individual pathogen level.

Previous studies have shown that pro-inflammatory cytokines and chronic inflammation are associated with cellular aging ("inflammaging") and a number of non-communicable diseases, including certain cancers, type 2 diabetes, and cardiovascular disease $(3,4,40,41)$. It would therefore not be surprising to find that infections also play a key role in these diseases and that the reactivation of these pathogens can contribute to the deterioration of the overall health of older individuals. Finally, CRP-PRS was also found to be significantly associated in the analysis including both genetics and serological results, confirming that human genetic variation plays a modulating role in systemic inflammation. 


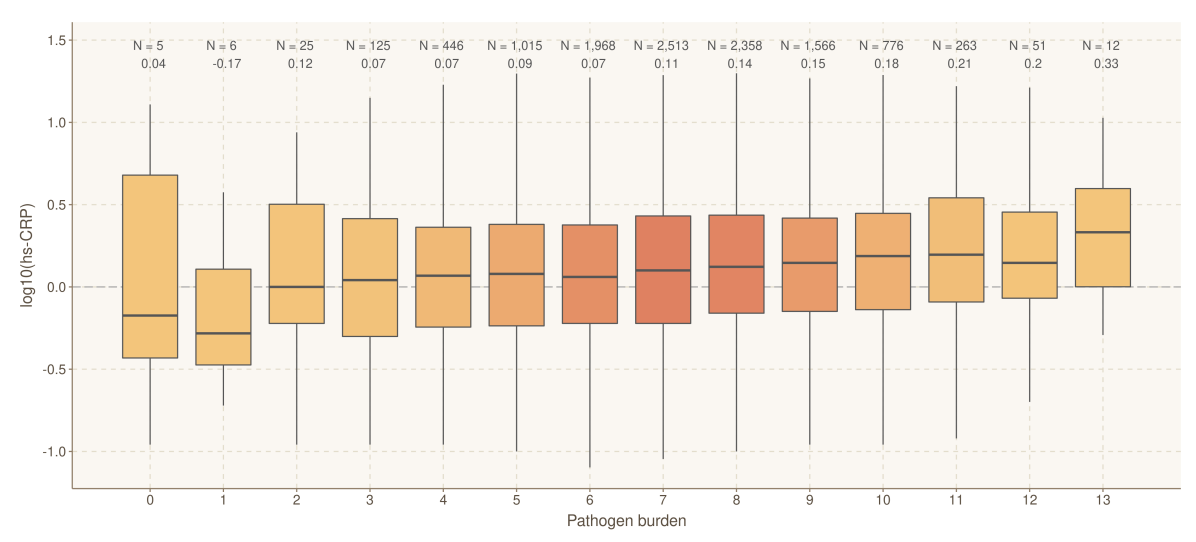

Fig. 4. Levels of hs-CRP by infectious burden. Boxplots showing the hs-CRP value for each pathogen load group. The black bold line within the boxplot indicates the median of the hs-CRP measurement. The boxes are colored by sample size. Sample size and median for each group are shown above the box.

Our study has some limitations. Firstly, we cannot rule out the effects of other non-measured infections at the time of hs-CRP measurement that may have influenced the level of inflammatory biomarker. Also, we did not adjust our models for all known influencing factors (e.g. smoking, antiinflammatory or anti-infective drugs, or possible inflammatory diseases). However, participants in both studies were assumed to be in good overall health at the time of data collection, and the data were filtered before analysis to detect levels indicative of acute infection. Secondly, some pathogens had relatively low or high seroprevalences and should be reexamined in a larger study. In particular, it will be interesting to repeat the analysis once serological data for all individuals in the UK Biobank are available. This will allow for greater reliability in terms of statistical power. Third, hs-CRP was the only inflammatory biomarker studied. Other proinflammatory cytokines such as IL- $1 \beta$, IL- 6 , and TNF- $\alpha$, are regulators of host responses to infection and positive mediators of inflammation. Consideration of these other biomarkers would give insight into more specific inflammatory pathways and provide a more comprehensive picture of the overall inflammatory status. Fourth, we only observed associations with the presence of chronic inflammation, and our study design does not allow us to infer any kind of causality. In particular, we can't exclude the possibility that higher levels of inflammation are responsible for the reactivation of a pathogen, resulting in detectable seropositivity.

In conclusion, we found that seropositivity for $C$. trachomatis and $H$. pylori antigens is associated with increased levels of hs-CRP. Together with demographic, clinical and genetic factors, persistent infections contribute to chronic low-grade inflammation, which can have deleterious long-term consequences on health.

\section{ACKNOWLEDGEMENTS}

We thank the participants in the UK Biobank and CoLaus|PsyCoLaus study for their time and contribution to this study. We also thank all the clinical, academic and administrative collaborators who helped with participant recruitment, study coordination, data collection and storage.

\section{AUTHOR CONTRIBUTIONS}

FH: Conceptualization, Methodology, Software, Formal analysis, Visualization, Writ- ing - Original Draft. ON: Formal Analysis. CB: Formal Analysis. NBr: Resources. NBe: Resources. TW: Investigation, Resources. PMV: Resources, Data Curation, Investigation. PV: Investigation. JF: Funding Acquisition, Project administration, Supervision, Writing - Original Draft. All co-authors reviewed the manuscript.

\section{CONFLICTS OF INTEREST}

The authors have no related financial or non-financial interests to disclose.

\section{FUNDING}

This project was supported by the Swiss National Science Foundation (grant 31003A_175603 to JF). The CoLaus|PsyCoLaus study was and is supported by research grants from GlaxoSmithKline, the Faculty of Biology and Medicine of Lausanne, and the Swiss National Science Foundation (grants 3200B0 105993, 3200B0_118308, 33CSCO_122661, 33CS30_139468, 33CS30_148401 and 33CS30_177535/1).

\section{DATA ACCESS}

The CoLaus|PsyCoLaus cohort data used in this study cannot be fully shared as they contain potentially sensitive patient information. As discussed with the competent authority, the Research Ethic Committee of the Canton of Vaud, transferring or directly sharing this data would be a violation of the Swiss legislation aiming to protect the personal rights of participants. Non-identifiable, individual-level data are available for interested researchers, who meet the criteria for access to confidential data sharing, from the CoLaus|PsyCoLaus Datacenter (CHUV, Lausanne, Switzerland). Instructions for gaining access to the CoLaus|PsyCoLaus data used in this study are available at (www.colaus-psycolaus.ch/professionals/how-tocollaborate/).

\section{Bibliography}

1. Ruslan Medzhitov. The spectrum of inflammatory responses. Science, 374(6571):10701075, 2021.

2. GB Ryan. Majno. acute inflammation: A review. Am J Pathol, 86:183-276, 1977.

3. Claudio Franceschi and Judith Campisi. Chronic inflammation (inflammaging) and its potential contribution to age-associated diseases. Journals of Gerontology Series A: Biomedical Sciences and Medical Sciences, 69(Suppl_1):S4-S9, 2014.

4. David Furman, Judith Campisi, Eric Verdin, Pedro Carrera-Bastos, Sasha Targ, Claudio Franceschi, Luigi Ferrucci, Derek W Gilroy, Alessio Fasano, Gary W Miller, et al. Chronic inflammation in the etiology of disease across the life span. Nature medicine, 25(12):18221832, 2019.

5. Carol A Feghali, Timothy M Wright, et al. Cytokines in acute and chronic inflammation. Front Biosci, 2(1):d12-d26, 1997.

6. Massimo De Martinis, Claudio Franceschi, Daniela Monti, and Lia Ginaldi. Inflammation markers predicting frailty and mortality in the elderly. Experimental and molecular pathology, 80(3):219-227, 2006.

7. Luigi Ferrucci, Annamaria Corsi, Fulvio Lauretani, Stefania Bandinelli, Benedetta Bartali, Dennis D Taub, Jack M Guralnik, and Dan L Longo. The origins of age-related proinflammatory state. Blood, 105(6):2294-2299, 2005.

8. Pedro Marques-Vidal, Murielle Bochud, François Bastardot, Thomas Lüscher, François Ferrero, Jean-Michel Gaspoz, Fred Paccaud, Adrian Urwyler, Roland von Känel, Christoph Hock, et al. Levels and determinants of inflammatory biomarkers in a swiss populationbased sample (colaus study). PloS one, 6(6):e21002, 2011.

9. Symen Ligthart, Ahmad Vaez, Urmo Võsa, Maria G Stathopoulou, Paul S De Vries, Bram P Prins, Peter J Van der Most, Toshiko Tanaka, Elnaz Naderi, Lynda M Rose, et al. Genome analyses of $>200,000$ individuals identify 58 loci for chronic inflammation and highlight pathways that link inflammation and complex disorders. The American Journal of Human $\mathrm{Ge}$ netics, 103(5):691-706, 2018. 
10. David H Thom, J Thomas Grayston, David S Siscovick, San-Pin Wang, Noel S Weiss, and Janet R Daling. Association of prior infection with chlamydia pneumoniae and angiographically demonstrated coronary artery disease. Jama, 268(1):68-72, 1992.

11. Jianhui Zhu, Arshed A Quyyumi, James E Norman, Gyorgy Csako, and Stephen E Epstein Cytomegalovirus in the pathogenesis of atherosclerosis: the role of inflammation as reflected by elevated c-reactive protein levels. Journal of the American College of Cardiology, 34(6):1738-1743, 1999.

12. Cathie Sudlow, John Gallacher, Naomi Allen, Valerie Beral, Paul Burton, John Danesh, Paul Downey, Paul Elliott, Jane Green, Martin Landray, et al. Uk biobank: an open access resource for identifying the causes of a wide range of complex diseases of middle and old age. PLoS medicine, 12(3):e1001779, 2015.

13. Mathieu Firmann, Vladimir Mayor, Pedro Marques Vidal, Murielle Bochud, Alain Pécoud, Daniel Hayoz, Fred Paccaud, Martin Preisig, Kijoung S Song, Xin Yuan, et al. The colaus study: a population-based study to investigate the epidemiology and genetic determinants of cardiovascular risk factors and metabolic syndrome. BMC cardiovascular disorders, 8(1): $1-11,2008$.

14. Clare Bycroft, Colin Freeman, Desislava Petkova, Gavin Band, Lloyd T Elliott, Kevin Sharp, Allan Motyer, Damjan Vukcevic, Olivier Delaneau, Jared O'Connell, et al. The uk biobank resource with deep phenotyping and genomic data. Nature, 562(7726):203-209, 2018.

15. Olivier Delaneau, Jonathan Marchini, and Jean-François Zagury. A linear complexity phasing method for thousands of genomes. Nature methods, 9(2):179-181, 2012.

16. Po-Ru Loh, Petr Danecek, Pier Francesco Palamara, Christian Fuchsberger, Yakir A Reshef Hilary K Finucane, Sebastian Schoenherr, Lukas Forer, Shane McCarthy, Goncalo R Abecasis, et al. Reference-based phasing using the haplotype reference consortium panel. Nature genetics, 48(11):1443-1448, 2016.

17. Shane McCarthy, Sayantan Das, Warren Kretzschmar, Olivier Delaneau, Andrew R Wood, Alexander Teumer, Hyun Min Kang, Christian Fuchsberger, Petr Danecek, Kevin Sharp, et al. A reference panel of 64,976 haplotypes for genotype imputation. Nature genetics, 48 (10): 1279,2016

18. Flavia Hodel, Amanda Y Chong, Petar Scepanovic, Zhi Ming Xu, Olivier Naret, Christian W Thorball, Sina Rüeger, Pedro Marques-Vidal, Peter Vollenweider, Martin Begemann, et al. Human genomics of the humoral immune response against polyomaviruses. Virus evolution, 7(2):veab058, 2021.

19. Tim Waterboer, Peter Sehr, Kristina M Michael, Silvia Franceschi, John D Nieland, Thomas $\mathrm{O}$ Joos, Markus F Templin, and Michael Pawlita. Multiplex human papillomavirus serology based on in situ-purified glutathione s-transferase fusion proteins. Clinical chemistry, 51(10):1845-1853, 2005.

20. Tim Waterboer, Peter Sehr, and Michael Pawlita. Suppression of non-specific binding in serological luminex assays. Journal of immunological methods, 309(1-2):200-204, 2006.

21. Alexander J Mentzer, Nicole Brenner, Naomi Allen, Thomas J Littlejohns, Amanda Y Chong Adrian Cortes, Rachael Almond, Michael Hill, Simon Sheard, Gil McVean, et al. Identification of host-pathogen-disease relationships using a scalable multiplex serology platform in uk biobank. medrxiv, page 19004960, 2019.

22. Christopher $C$ Chang, Carson $C$ Chow, Laurent CAM Tellier, Shashaank Vattikuti, Shaun $M$ Purcell, and James $\mathrm{J}$ Lee. Second-generation plink: rising to the challenge of larger and richer datasets. Gigascience, 4(1):s13742-015, 2015.

23. Kyoko Watanabe, Erdogan Taskesen, Arjen Van Bochoven, and Danielle Posthuma. Functional mapping and annotation of genetic associations with fuma. Nature communications, 8(1):1-11, 2017.

24. GTEx Consortium et al. The gtex consortium atlas of genetic regulatory effects across human tissues. Science, 369(6509):1318-1330, 2020.

25. Bruce M Psaty, Christopher J O'donnell, Vilmundur Gudnason, Kathryn L Lunetta, Aaron R Folsom, Jerome I Rotter, André G Uitterlinden, Tamara B Harris, Jacqueline CM Witteman, and Eric Boerwinkle. Cohorts for heart and aging research in genomic epidemiology (charge) consortium: Design of prospective meta-analyses of genome-wide association studies from 5 cohorts. Circulation: Cardiovascular Genetics, 2(1):73-80, 2009.

26. Shing Wan Choi, Timothy Shin-Heng Mak, and Paul F O'Reilly. Tutorial: a guide to performing polygenic risk score analyses. Nature Protocols, 15(9):2759-2772, 2020.

27. R Core Team et al. R: A language and environment for statistical computing. 2013.

28. Abbas Dehghan, Josée Dupuis, Maja Barbalic, Joshua C Bis, Gudny Eiriksdottir, Chen Lu, Niina Pellikka, Henri Wallaschofski, Johannes Kettunen, Peter Henneman, et al. Metaanalysis of genome-wide association studies in $>80000$ subjects identifies multiple loci for c-reactive protein levels. Circulation, 123(7):731-738, 2011.

29. Bram P Prins, Karoline B Kuchenbaecker, Yanchun Bao, Melissa Smart, Delilah Zabaneh, Ghazaleh Fatemifar, Jian'an Luan, Nick J Wareham, Robert A Scott, John RB Perry, et al. Genome-wide analysis of health-related biomarkers in the uk household longitudinal study reveals novel associations. Scientific reports, 7(1):1-9, 2017.

30. Alexander P Reiner, Mathew J Barber, Yongtao Guan, Paul M Ridker, Leslie A Lange, Daniel I Chasman, Jeremy D Walston, Gregory M Cooper, Nancy S Jenny, Mark J Rieder, et al. Polymorphisms of the hnf1a gene encoding hepatocyte nuclear factor-1 $\alpha$ are associated with c-reactive protein. The American Journal of Human Genetics, 82(5):1193-1201, 2008.

31. Paul M Ridker, Guillaume Pare, Alex Parker, Robert YL Zee, Jacqueline S Danik, Julie E Buring, David Kwiatkowski, Nancy R Cook, Joseph P Miletich, and Daniel I Chasman. Loci related to metabolic-syndrome pathways including lepr, hnf1a, il6r, and gckr associate with plasma c-reactive protein: the women's genome health study. The American Journal of Human Genetics, 82(5):1185-1192, 2008.

32. Christian Hammer, Martin Begemann, Paul J McLaren, István Bartha, Angelika Michel, Beate Klose, Corinna Schmitt, Tim Waterboer, Michael Pawlita, Thomas F Schulz, et al. Amino acid variation in hla class ii proteins is a major determinant of humoral response to common viruses. The American Journal of Human Genetics, 97(5):738-743, 2015.

33. Petar Scepanovic, Cécile Alanio, Christian Hammer, Flavia Hodel, Jacob Bergstedt, Etienne Patin, Christian W Thorball, Nimisha Chaturvedi, Bruno Charbit, Laurent Abel, et al. Human genetic variants and age are the strongest predictors of humoral immune responses to common pathogens and vaccines. Genome medicine, 10(1):1-13, 2018.

34. P Sipponen and H Hyvärinen. Role of helicobacter pylori in the pathogenesis of gastritis, peptic ulcer and gastric cancer. Scandinavian Journal of Gastroenterology, 28(sup196): 3-6, 1993.

35. D Taylor-Robinson and BJ Thomas. The rôle of chlamydia trachomatis in genital-tract and associated diseases. Journal of Clinical Pathology, 33(3):205, 1980.

36. Louisa Jackson, John Britton, Sarah A Lewis, Tricia M McKeever, John Atherton, Donna Fullerton, and Andrew W Fogarty. A population-based epidemiologic study of helicobacter pylori infection and its association with systemic inflammation. Helicobacter, 14(5):460-465, 2009.

37. Preeti Dubey, Kiran Pandey, Neetu Singh, Ajay Bhagoliwal, and Deepti Sharma. Role of maternal serum chlamydia trachomatis igg antibodies and serum c-reactive protein in preterm labor. Int J Reprod Contracept Obstet Gynecol, 3(1):195-198, 2014

38. JE Den Hartog, JA Land, FRM Stassen, AGH Kessels, and CA Bruggeman. Serological markers of persistent c. trachomatis infections in women with tubal factor subfertility. Human reproduction, 20(4):986-990, 2005.

39. Liisa Karinen, Anneli Pouta, Aini Bloigu, Pentti Koskela, Mika Paldanius, Maija Leinonen, Pekka Saikku, Marjo-Riitta Jêrvelin, and Anna-Liisa Hartikainen. Serum c-reactive protein and chlamydia trachomatis antibodies in preterm delivery. Obstetrics \& Gynecology, 106 (1):73-80, 2005.

40. Gaëtan Gavazzi and Karl-Heinz Krause. Ageing and infection. The Lancet infectious diseases, 2(11):659-666, 2002

41. Nemat Khansari, Yadollah Shakiba, and Mahdi Mahmoudi. Chronic inflammation and oxidative stress as a major cause of age-related diseases and cancer. Recent patents on inflammation \& allergy drug discovery, 3(1):73-80, 2009. 